\title{
Los Working Papers al servicio de la escritura productiva
}

\author{
Juan A. Marin-Garcia' ${ }^{1}$, Jose P. Garcia-Sabater ${ }^{1}$
}

${ }^{1}$ ROGLE. Dpto. de Organización de Empresas. Universidad Politécnica de Valencia. Camino de Vera S/N
46022 Valencia. jamarin@omp.upv.es, jpgarcia@omp.upv.es

Abstract:

- Purpose of the paper: describir el papel de los Working Papers y cómo usarlos para divulgar cápsulas de conocimiento que faciliten el proceso de coordinación, aprendizaje interno y la escritura de artículos de investigación.

Keywords: Escritura productiva, cápsulas de conocimiento,

\section{Función y formato de los Working Papers}

El objetivo de los Working Papers (WP) es facilitar el acceso al debate académico de avances y resultados de trabajos de investigación, así como de fragmentos de investigación que posteriormente se convertirán en artículos científicos. De este modo, se pueden citar y proteger frente a plagio las ideas escritas en versiones preliminares, sin menoscabo de ser divulgadas en otros medios o publicaciones que el autor considere adecuadas. Con ello se pretende dar soporte a la comunicación científica facilitando el acceso con garantías a la publicación académica en revistas consolidadas, por medio de:

- Rodaje en el sistema de publicación académica

- Debate de las ideas presentadas

- Construir progresivamente documentos más complejos

- Fijar fechas de entrega intermedias para dinamizar la actividad de doctorandos y miembros del grupo de investigación

- Revisar y pulir fragmentos de comunicación en momentos cercanos a su creación

- Generar un diario/portafolio de investigación -se pueden ver detalles de lo que es un diario de investigación en Primo Yúfera (1994), páginas 136-151.

Una posible función de los WP (figura 1) consiste en ir completando los documentos complejos por medio de partes que sean autónomas. De este modo se pretende reducir el tiempo necesario en el proceso de escritura científica, manteniendo o incluso incrementando la calidad del documento (Morss y Murray, 2001). Por ejemplo, una comunicación a un congreso se puede construir integrando diferentes WP. En cada uno de ellos se ha abordado y resuelto una parte de la línea argumental, de hecho uno puede ser la manifestación de las preguntas de investigación, o la línea argumental, o la justificación de la pertinencia o viabilidad académica de esa línea argumental o los diferentes caminos para abordar un tema y las dudas que genera el no saber cuál de ellos decidir. La comunicación a un congreso, probablemente deje algunas lagunas sin cerrar completamente. Por ejemplo, algunas afirmaciones sin suficientes citas que las avalen, o algunos resultados que no se sabe cómo explicar en discusión o algunas partes sin suficiente revisión bibliográfica pues se trata de descubrimientos realizados sobre la marcha o algunos comentarios de los revisores que necesitan ser trabajados. Los WP se pueden utilizar para realizar estas tareas y como resultado, poder crear la versión de un artículo para una revista académica. En todo este proceso será necesario enlazar los contenidos de los WP. Normalmente no será posible copiar y pegar uno tras otro, sino que habrá que transformarlos para encajarlos en un documento conjunto. 


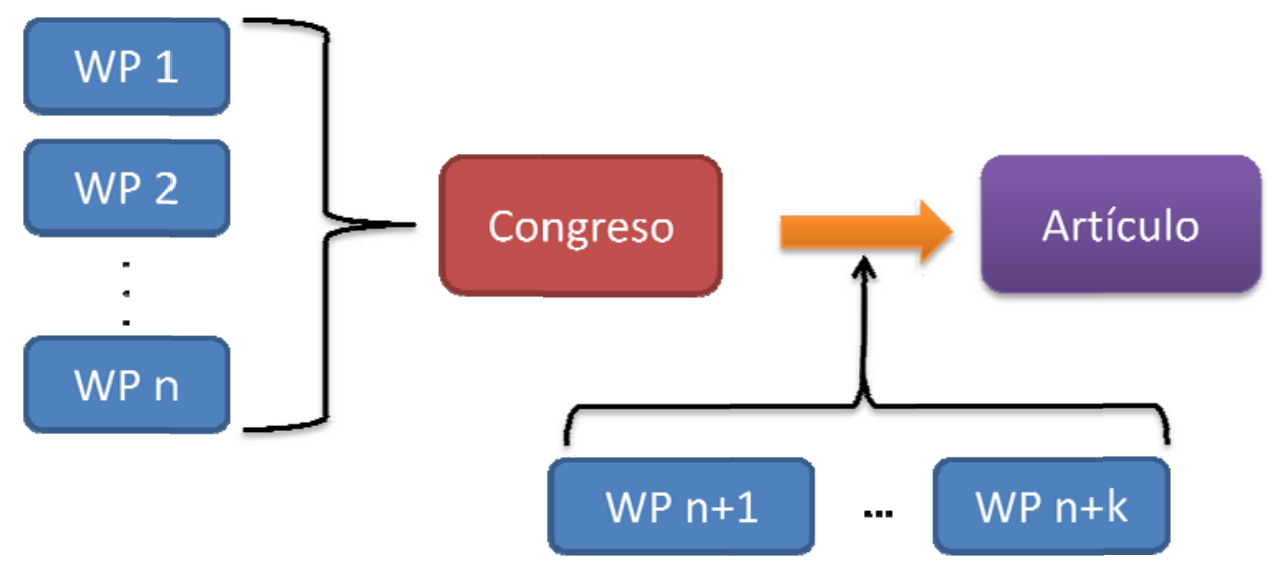

Figura 1. Integración de los WP. Fuente: elaboración propia

Se pretende, por tanto, que los WP constituyan por sí mismos una contribución científica, pequeña pero suficiente para ser comprendida, valorada y discutida sin necesidad de consultar otros documentos adicionales. En otras palabras, que los WP sean unas cápsulas de conocimiento (CC) (figura 2).

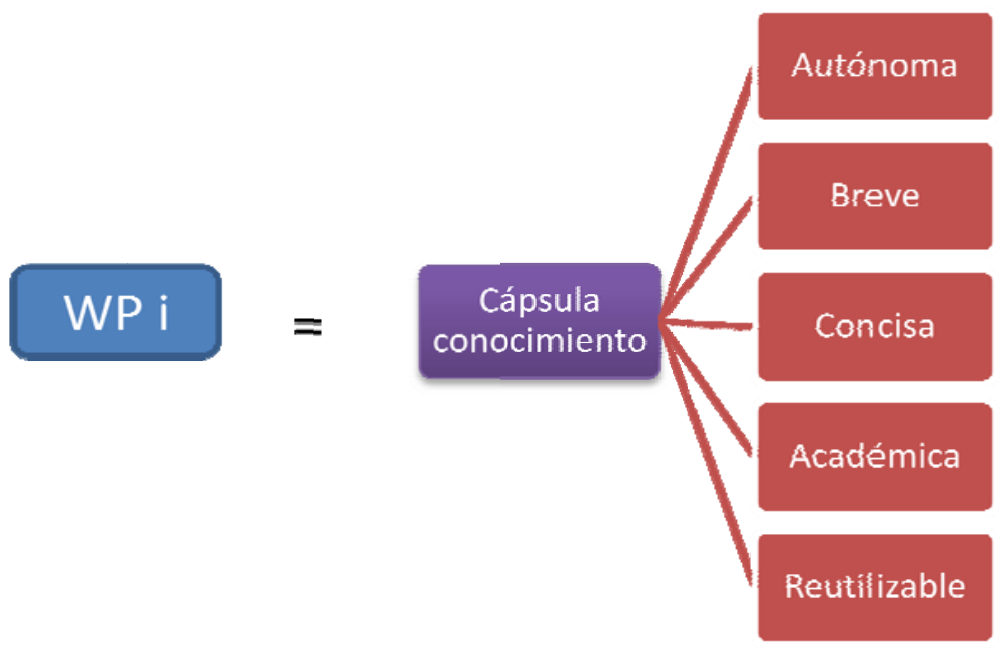

Figura 2. Cápsulas de conocimiento. Fuente: elaboración propia

El objeto de las cápsulas de conocimiento es estandarizar la comunicación de pequeños paquetes de conocimiento que cuestan alrededor de unas 40 horas de trabajo de generar (incluyendo la definición, lectura de bibliografía, escritura, etc.). La idea es que cada cápsula contenga entre 2 y 6 páginas. Por ejemplo, una sección del marco teórico acabada (poniéndole una introducción y unas conclusiones muy breves que permitan valorarlo en su contexto). Como es posible que sean reutilizadas en documentos más extensos, deben tener el mínimo formato de párrafos o de tablas, para que luego sea fácil adaptarla a las exigencias editoriales del documento definitivo. Cada cápsula de conocimiento debe contener bibliografía insertada y formateada con Reference Manager (existen otros gestores de bibliografía pero no son tan buenos, y, en cualquier caso, este es el estándar del grupo ROGLE). Para cumplir con las normas habituales en muchas revistas académicas, es conveniente que las frases se construyan utilizando la forma reflexiva. Es decir se escribirá "se escribirá" y no "escribiremos" o "escribiré" (Marin-Garcia, 2008; Valderrama, 2005). Esto es discutible, pues algunas revistas no imponen esta limitación, pero otras sí. Los autores de esta comunicación no se han 


\section{Working Papers on Operations Management}

encontrado todavía una revista que exija que se use la primera persona del singular o plural en lugar de la forma impersonal o reflexiva. Por eso se hace la recomendación que permite superar las normas de estilo de las revistas más “intrusivas”.

Las principales características que debe cumplir una cápsula de conocimiento del grupo ROGLE son (figura 2):

- Autónoma: cada cápsula aporta los elementos de juicio indispensables para que el lector pueda situarse ante un tema determinado. Se pueden comprender por sí mismas y están escritas con un estilo claro.

- Breves: se pueden leer y revisar rápidamente pues no tienen una extensión grande

- Concisa: se centran en realizar sólo una contribución académica minúscula pero significativa. Son la mínima expresión de los granos de arena que acaban construyendo la montaña del conocimiento.

- Académica: respetan las exigencias de la comunicación académica (rigor, replicación, justificación...) (Marin-Garcia, 2008).

- Reutilizable: constituyen un conocimiento modular, sin apenas formato y con las citas embebidas. Por todo ello, se pueden reutilizar en diferentes contextos, exigiendo una mínima adaptación.

\section{Proceso de escritura y publicación de un WP}

De manera esquemática, la publicación de un WP debería pasar por las siguientes fases:

- Se busca un objetivo (que se especifica en el resumen del WP).

- Se lee y ficha el material disponible para el objetivo (figura 3).

- Se procesan las fichas para construir el WP (manifiestas lo que tienes claro y las cosas que te plantean dudas).

- Se enlaza en la introducción con lo que sea necesario para comprenderlo en conjunto (si es necesario se citan otros WP).

- Se debate en grupo y se somete a proceso de revisión ciega.

- Los comentarios de los revisores se integran en el documento que se remite de nuevo para la publicación.

Si el documento no constituye una contribución autónoma o modular, será rechazado indicando qué cosas faltan. Por lo tanto, los rechazos son una manera de mejorar y pulir los documentos y deben valorarse como críticas constructivas que permiten reconducir la investigación y ahorrar tiempo de desarrollo de comunicaciones científicas.

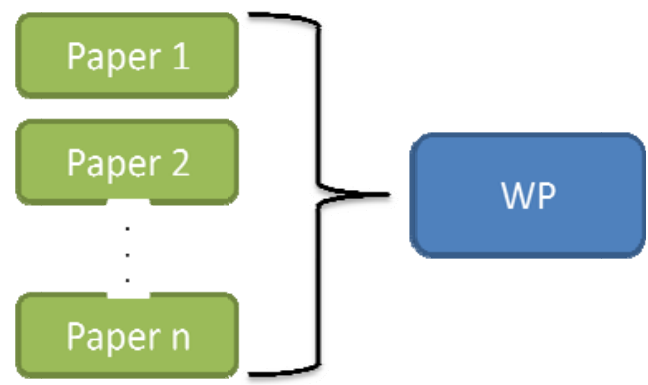

Figure 3. Los WP se nutren de artículos científicos 


\section{Posibles extensiones de este WP}

Se podrían considerar las siguientes líneas de trabajo futuras, relacionadas con este documento:

1. Cómo escribir contribuciones científicas: una extensión de Marin-Garcia (2008) adatada a las particularizaciones concretas de cada tipo de investigación, por ejemplo estudio de casos, action research, encuestas, etc. (Bonache, 1999; Chabaud y Germain, 2006; Friedman y Rogers, 2009; Frohlich, 2002; Gibbert et al., 2008; Gupta et al., 2007; Huxham y Vangen, 2003; Langley y Royer, 2006; McCutcheon y Meredith, 1993; Piekkari et al., 2009; Shah y Goldstein, 2006; Synodinos, 2003; Voss et al., 2002; Yin, 1994).

2. El papel de los WP al servicio de la transferencia de investigación a los profesionales

3. Funciones y desarrollo de las exposiciones orales de WP en Jornadas de investigación. Aplicación a las tesis doctorales, a la construcción de cápsulas de conocimiento, etc.

4. Construcción de una parrilla de evaluación de cápsulas de conocimiento (en su versión CC_académica y CC_profesional y otras versiones si fuese necesario, por ejemplo, RevisorDoctorando). La idea es que estas plantillas sean una ayuda al revisor para facilitarle la tarea -que se centre en lo que es realmente importante y que no tenga que teclear demasiado- y que proporcionen un feedback útil para el autor les de orientación de en qué deben centrarse al escribir o qué aspectos deben tener en cuenta para mejorar.

\section{Referencias.}

Bonache, J. (1999). El estudio de casos como estrategia de construcción teórica: características, críticas y defensas. Cuadernos de Economía y Dirección de la Empresa, Vol. 3, pp. 123-140.

Chabaud, D.; Germain, O. (2006). La réutilisation de données qualitatives en sciences de gestion : un second choix ? M@n@gement, Vol. 9, nº. 3, pp. 191-213.

Friedman, V. J.; Rogers, T. (2009). There is nothing so theoretical as good action research. Action Research, Vol. 7, no. 1, pp. 31-47.

Frohlich, M. T. (2002). Techniques for improving response rates in OM survey research. Journal of Operations Management, Vol. 20, nº 1, pp. 53-62.

Gibbert, M.; Ruigrok, W.; Wicki, B. (2008). Research notes and comentaries. Waht passes as a rigorous case study? Strategic Management Journal, Vol. 29, pp. 1465-1474.

Gupta, S.; Verma, R.; Victorino, L. (2007). Empirical Research Published in Production and Operations Management (1992-2005): Trends and Future Research Directions. Production and Operations Management, Vol. 15, $\mathrm{n}^{\circ}$. 3, pp. 432-448.

Huxham, C.; Vangen, S. (2003). Researching Organizational Practice through Action Research: Case Studies and Design Choices. Organizational Research Methods, Vol. 6, $\mathrm{n}^{\circ}$. 3, pp. 383-403.

Langley, A.; Royer, I. (2006). Perspectives on Doing Case Study Research in Organizations. M@n@gement, Vol.9, nº. 3, pp. 73-86.

Marin-Garcia, J. A. (2008). Guía de investigación para el desarrollo de la carrera del profesorado: organización de empresas (volumen 13). Editorial de la Universidad Politécnica de Valencia.

McCutcheon, D. M.; Meredith, J. R. (1993). Conducting case study research in operations management. Journal of Operations Management, Vol. 11, nº. 3, pp. 239-256.

Morss, K.; Murray, R. (2001). Researching Academic Writing within a Structured Programme: insights and outcomes. Studies in Higher Education, Vol. 26, nº. 1, pp. 35-52. 
Piekkari, R.; Welch, C.; Paavilainen, E. (2009). The Case Study as Disciplinary Convention: Evidence From International Business Journals. Organizational Research Methods, Vol. 12, $n^{\circ} .3$, pp. 567-589.

Primo Yúfera, E. (1994). Introducción a la investigación científica y tecnológica. Alianza universidad.

Shah, R.; Goldstein, S. M. (2006). Use of structural equation modeling in operations management research: Looking back and forward. Journal of Operations Management, Vol. 24, no. 2, pp. 148-169.

Synodinos, N. E. (2003). The "art" of questionnaire construction: Some important considerations for manufacturing studies. Integrated Manufacturing Systems, Vol. 14, $\mathrm{n}^{\mathrm{o}}$. 3, pp. 221-237.

Valderrama, J. O. (2005). Principales Aspectos sobre la Preparación de un Artículo para ser Publicado en una Revista Internacional de Corriente Principal. Revista Internacional de Informacion Tecnologica, Vol. 16, $\mathrm{n}^{\circ}$. 2, pp. 3-14.

Voss, C.; Tsikriktsis, N.; Frohlich, M. (2002). Case research in operations management. International Journal of Operations \& Production Management, Vol. 22, nº. 2, pp. 195-216.

Yin, R. (1994). Case study research, 2nd ed. Sage. 